\title{
Techniques de visualisations du déferlement en canal à houle
}

\author{
Christophe Chevalier \\ Docteur, M2C-Groupe Mécanique des Fluides, Université de Caen \\ Dominique Mouazé \\ Doctorant, M2C - Groupe Mécanique des Fluides, Université de Caen
}

\begin{abstract}
Résumé
L'objet de cette communication est la présentation de résultats de visualisations mettant en valeur différentes techniques employées en laboratoire. Ces séances permettent en particulier de mieux appréhender les mécanismes du déferlement d'une houle régulière et d'illustrer le comportement tourbillonnaire autour d'un cylindre près du fond.
\end{abstract}

\begin{abstract}
The object of this communication is the presentation of results of visualizations emphasizing various techniques employed in the laboratory. These experiments make it possible in particular to better apprehend the mechanisms of the breaking of a regular wave and to illustrate the vortex behavior around a cylinder close to the bottom.
\end{abstract}

\section{Introduction}

Les visualisations représentent un des outils expérimentaux les plus intéressants de la mécanique des fluides car elles permettent de rendre directement accessible à la perception visuelle certaines propriétés de l'écoulement. Dans le domaine du génie côtier, les visualisations sont de plus en plus répandues même si les écoulements de type houle et les installations expérimentales utilisées peuvent rendre la mise en œuvre de telles expérimentations plus difficiles.

L'étude du déferlement est l'un des sujets de recherche en génie côtier où les visualisations sont les plus répandues et parmi les spectaculaires. L'objet de cet article est la présentation de quelques résultats obtenus en laboratoire sur ce sujet à l'aide de différentes techniques de visualisations complémentaires.

\section{Dispositif expérimental}

Lors de la propagation de la houle vers la côte, l'action conjuguée des phénomènes de réfraction (diminution de la longueur d'onde) et de shoaling (augmentation de la hauteur de vague) conduit à une augmentation de la cambrure de vague jusqu'à la limite de stabilité où la houle déferle. C'est un phénomène très spectaculaire au cours duquel l'onde est partiellement ou totalement détruite (voir Figure 1). Il est caractérisé par un haut degré de turbulence et une grande dissipation d'énergie. 
On distingue classiquement trois grands types de déferlement :

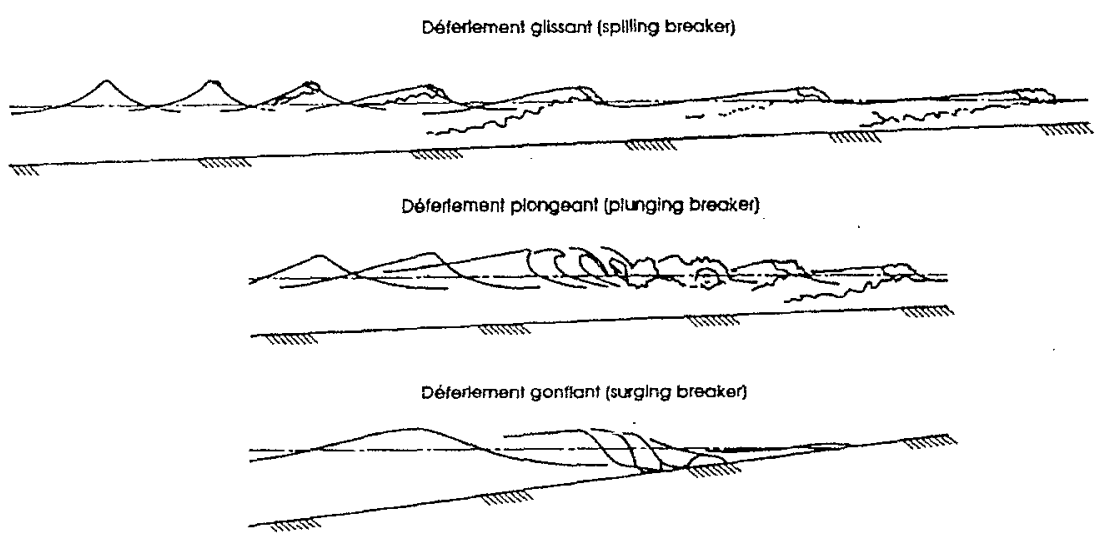

Schémas des différents déferlements d'après Svendsen (dans Dean et Dalrymple, 1991)

- déferlement glissant

Il est caractérisé par l'apparition d'écume et de turbuience au sommet de la houle avec des bulles d'air qui glissent sur le front aval, recouvrant celui-ci. Le profil est à peine dissymétrique. Le déferlement glissant débute lorsqu'une partie de la crête se déplace plus rapidement que le reste de la vague en "glissant" sur le front aval en un mélange eau - air. Ensuite, la lame évolue vers la formation d'un petit raz de marée ou mascaret. L'énergie est dissipée graduellement à l'approche de la plage. Ce type de déferlement apparait lorsque la pente de la plage est généralement très faible et le profil de la vague très cambré.

- déferlement plongeant (voir Figure 5 et Figure 6)

Pour ce type de déferlement, les lames prennent rapidement une forme dissymétrique avec une face antérieure concave, parfaitement lisse et presque verticale que la crête finit par surplomber. Il y a alors projection du sommet de la houle vers l'aval et formation d'un tube d'eau à l'intérieur duquel l'air se trouve emprisonné. Lorsque la crête retombe brutalement en nappe déversante, la lame dégénère alors en une onde de ressaut se propageant jusqu'au rivage. Ce type de déferlement se produit pour des pentes de plage supérieures au cas du déferlement glissant mais restant assez faibles.

Ce déferlement est le plus spectaculaire. Caractérisé par le bruit du plongeon de la crête, c'est aussi celui présentant à priori une zone de turbulence plus développée.

- déferlement gonflant

Le front de l'onde et la crête sont relativement peu déformés et la vague se propage sur la plage avec une faible formation d'écume et de bulles. Ce déferlement s'apparente à une onde stationnaire. Il est caractéristique des houles de faibles cambrures et de pente plus importante que pour les cas précédents.

Figure 1 : Les différents types de déferlement Different breaker types

Pour obtenir le déferlement d'une vague régulière, nous avons mis en place un fond en pente afin de provoquer le déferlement par diminution de la profondeur locale. 
Les principaux éléments constitutifs de ce dispositif expérimental sont les suivants (Figure 2):

- Le canal à houle du laboratoire (22 $\mathrm{m}$ de long pour $0,8 \mathrm{~m}$ de large, profondeur d'eau au cours des essais comprise entre 0,4 et $0,5 \mathrm{~m}$ ). Ce canal est équipé d'un batteur type piston permettant de générer des houles régulières et réelles. Les parois latérales du canal sont vitrées de même que 3 éléments du fond afin de faciliter les visualisations mais aussi les mesures par Vélocimétrie Doppler Laser VDL.

- La plage en PVC de faible pente $(5 \%)$ de longueur $10 \mathrm{~m}$. Cette plage est réalisée en 5 éléments modulables de $2 \mathrm{~m}$ chacun. Un de ceux-ci comprend un fond transparent. En positionnant celui-ci sur une partie du canal à fond vitré, il est ainsi possible de procéder à un éclairage de l'écoulement (par spot stroboscopique ou plan laser) par-dessous la veine d'essai.

Par ailleurs, l'instrumentation classique de la surface libre par des sondes résistives permet de définir les caractéristiques des houles étudiées.

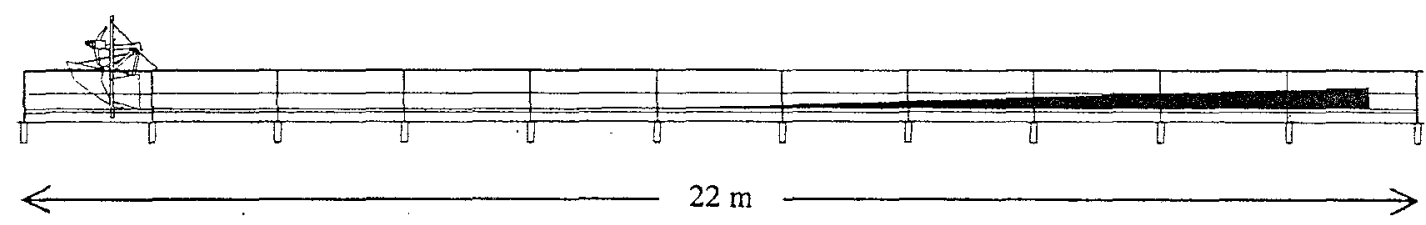

Figure 2 : Dispositif expérimental pour la génération de houles déferlantes Experimental set-up for breaking waves generation

Deux principaux types de visualisations ont été réalisés au cours de cette étude (déferlement, écoulement autour du cylindre). Ces deux types reposent sur du matériel classique de prise de vue :

- Pour les prises de vues vidéo, nous avons utilisé un caméscope Sony HI-8 mm (acquisition standard à 25 images/s). L'acquisition vidéo donne une vision dynamique des phénomènes complexes de l'écoulement, en particulier lors de la phase de déferlement, ainsi que le mouvement et la combinaison des tourbillons autour du cylindre ;

- Les appareils photographiques utilisés sont des reflex $24 \times 36 \mathrm{~mm}^{2}$ permettant des prises de vue en rafales (Nikon F 801 à 3,3 images/s maximum et Canon EOS 50 à 2,5 images/s maximum).

Ces deux moyens sont parfaitement complémentaires. Ainsi, la fréquence d'acquisition est moins rapide pour les photos mais la qualité de prise de vues est meilleure (résolution). Par ailleurs, la vidéo peut être visionnée directement sur écran au cours des essais alors que les photographies doivent être développées, rendant l'analyse de l'expérience moins immédiate.

\section{Visualisations du déferlement}

Au cours de nos "essais, il a été possible d'obtenir les 3 types existants (glissant, plongeant et gonflant). Cependant, la grande majorité des déferlements réalisés 
était de type glissant ou plongeant. Ceci s'explique par la pente intermédiaire étudiée et la gamme de périodes utilisée (entre 1,5 et 2,5 s).

Différentes prises de vues (vidéo ou photos) ont ainsi pu être réalisées illustrant le processus de déferlement. Nous montrons ici des illustrations obtenues par des prises de vues classiques (avec un éclairage traditionnel) des structures tourbillonnaires après le point de déferlement ainsi que des exemples de visualisations obtenues sous éclairage stroboscopique.

\subsection{Mise en évidence des structures tourbillonnaires}

Le déferlement induit des tourbillons qui jouent notamment un rôle important dans la remise en suspension des sédiments dans la zone de surf. Nadaoka et al. (1989) ont ainsi mis en évidence par des visualisations de l'écoulement des structures tourbillonnaires d'axes verticales obliques lors d'un déferlement glissant. Cette observation est confirmée par Zhang et Sunamura (1994) et deux nouveaux types de tourbillons d'axe horizontal sont également définis suivant le type de déferlement (Figure 3).

Ces différents types de tourbillons ont également été observés au cours de nos expériences. La Figure 4 présente ainsi deux photos successives d'un déferlement plongeant montrant la présence de ces structures tourbillonnaires d'axes obliques et horizontales de type A.
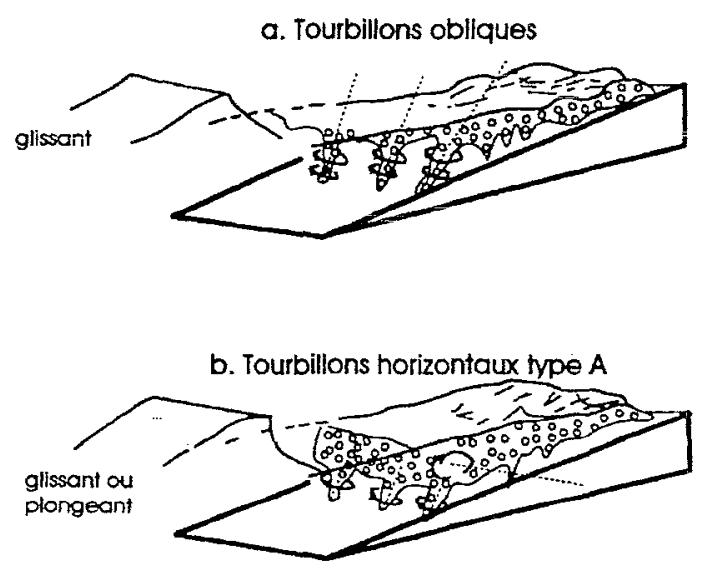

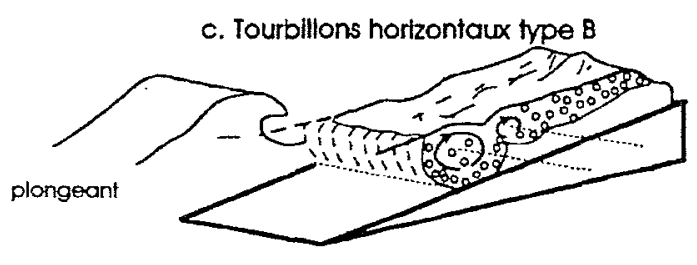

Figure 3 : Les trois types de tourbillons selon Zhang et Sunamura (1994)

Three vortex types according to Zhang et Sunamura (1994)

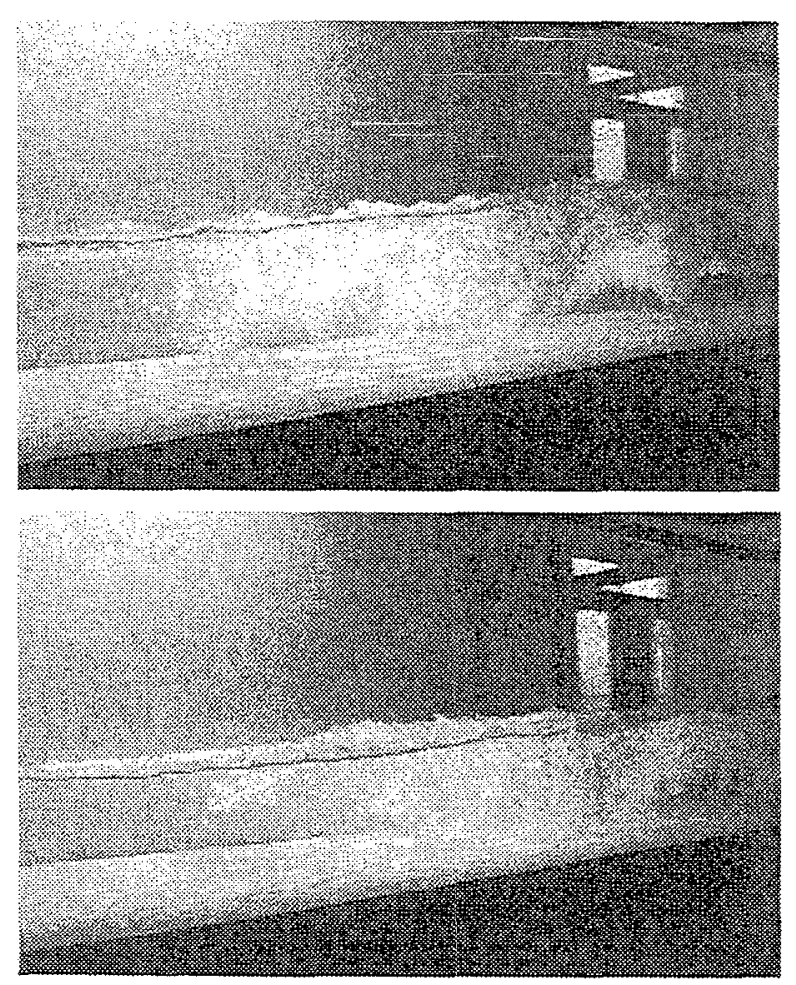

Figure 4 : Mise en évidence de tourbillons obliques et horizontaux type $\mathrm{A}$

Highlighting of oblique vortex and A-type horizontal vortex 


\subsection{Visualisations par éclairage stroboscopique}

Dans le cas d'un phénomène dynamique rapide comme le déferlement, un éclairage stroboscopique est particulièrement intéressant puisqu'il permet de décomposer différentes phases du mouvement lors de l'observation. Associé à une prise de vue adéquate, il est ainsi possible de réaliser des photographies spectaculaires de la phase de déferlement.

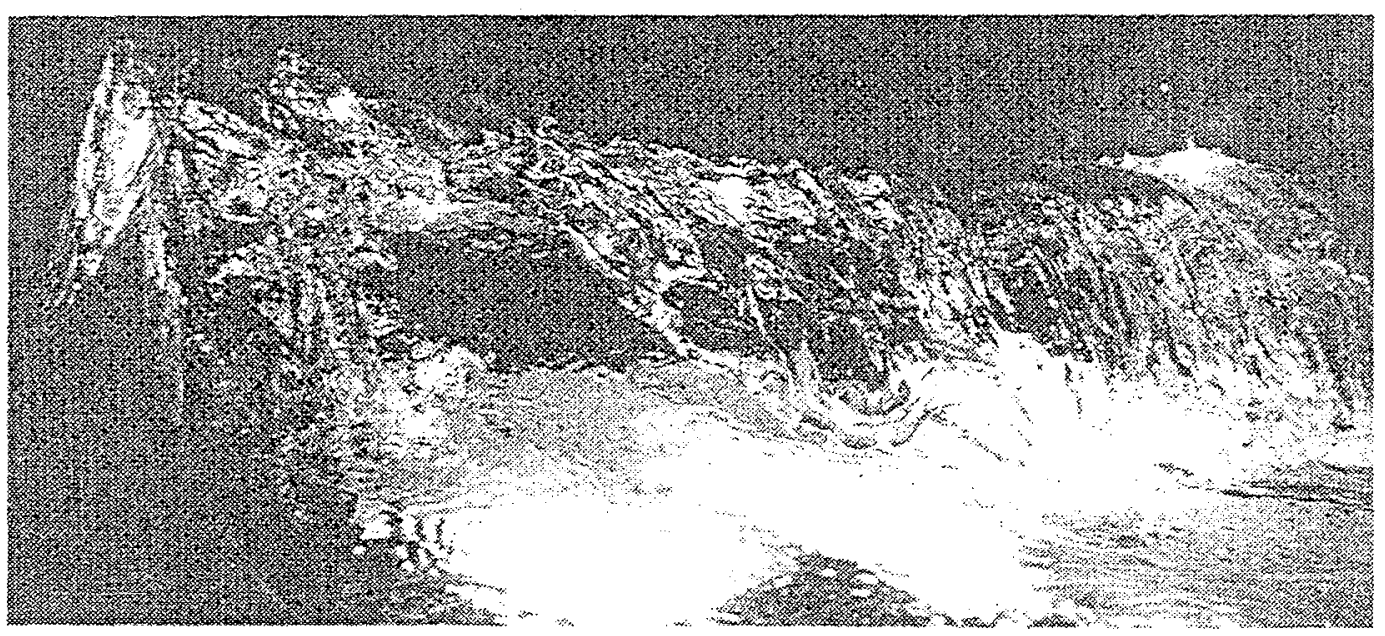

Figure 5 : Prise de vue avec éclairage complémentaire par stroboscope Picture taken using complementary stroboscope lighting

La photographie de la Figure 5 illustre le cas d'utilisation du stroboscope comme flash additionnel déporté. Le dispositif d'éclairage est positionné sous le canal. En faisant le choix d'une pose longue sur l'appareil photo et d'une fréquence de déclenchement faible de l'éclairage stroboscopique (correspondant à un seul éciat pendant le temps de prise de vue), il est ainsi possible de figer le mouvement. On profite ainsi d'une puissance d'éclairage bien supérieure aux flashs traditionnels. L'inconvénient résulte dans la difficulté à maîtriser le temps de déclenchement de l'éclair.

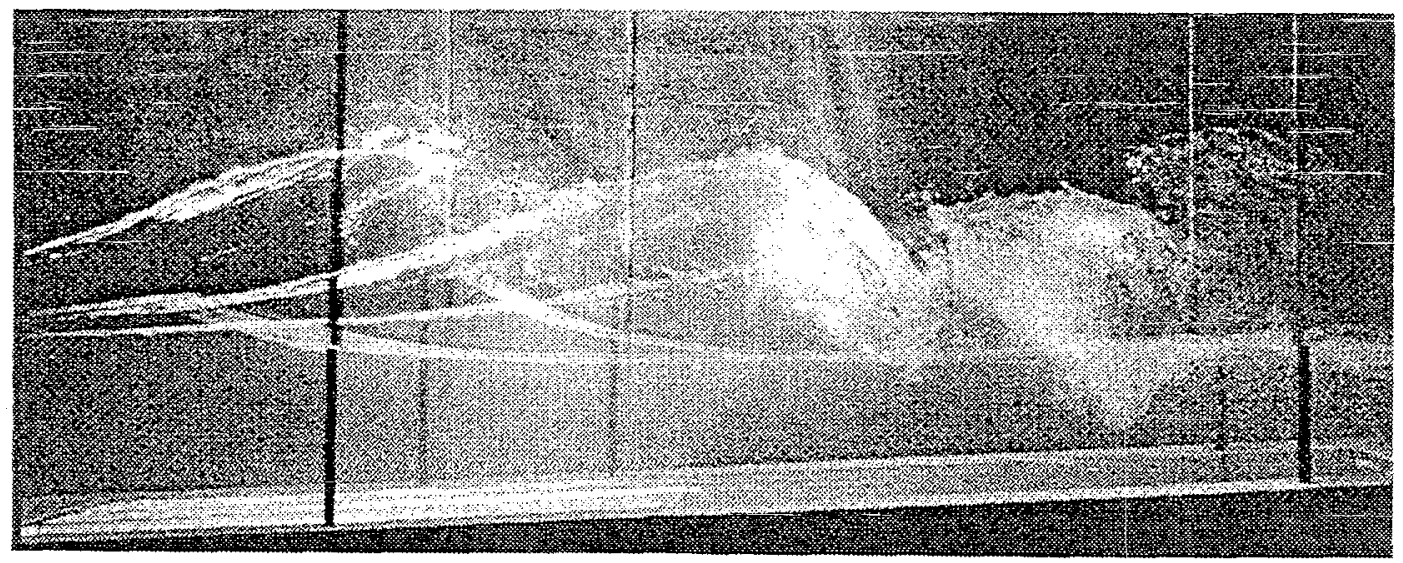

Figure 6 : Déferlement plongeant sous éclairage stroboscopique multiple Plunging breaker under multiple stroboscopic lighting 
La Figure 6 est un exemple de prise de vue comprenant plusieurs éclats de stroboscope (temps de pose long). Ceci permet de superposer différentes phases de l'écoulement et en particulier d'observer l'évolution de la surface libre autour du point de déferlement. On peut ainsi noter une rapide diminution de la crête de vague lors de ce déferlement plongeant.

\section{Application à l'étude de l'écoulement autour d'un cylindre en zone côtière}

Le but de ces visualisations est l'étude de l'écoulement de houle autour d'un cylindre (diamètre $\mathrm{D}=4 \mathrm{~cm}$ ) à proximité du fond incliné de pente égale à $5 \%$. La mise en œuvre de visualisations dans le canal à houle est principalement motivée par le souci de mieux comprendre l'écoulement généré autour du cylindre. Ainsi cette méthode qualitative noús donne une vision globale des phénomènes autant sur le point de vue spatial (évolution des zones tourbillonnaires, estimation des points de décollement, zones de hautes vitesses) que sur le plan temporel (dynamique de l'écoulement, périodicité des événements, instabilités).

Différentes configurations géométriques ont été testées $(0<\mathrm{e} / \mathrm{D}<1)$ pour des houles de peu déformées à limite déferlantes, soit $4<\mathrm{KC}<26$, où $\mathrm{KC}$ est le nombre de Keulegan et Carpenter :

$$
\mathrm{KC}=\mathrm{U}_{\max } \mathrm{T} / \mathrm{D}
$$

$T$ est la période de la houle incidente, $U_{\max }$ la vitesse horizontale maximale de l'écoulement libre au niveau du cylindre et e l'écartement de la génératrice inférieure du cylindre par rapport au fond.

\subsection{Visualisations par nappe laser}

Une méthode courante de visualisation de l'écoulement est d'ensemencer celui-ci à l'aide de très fines particules et d'éclairer-le tout par une nappe laser verticale, perpendiculaire à l'axe de prise de vue (Merzkirch, 1987). Suivant ce principe, nous avons procédé à des visualisations de l'écoulement autour d'un cylindre en utilisant le module transparent inséré dans le plage de déferlement (caisson également employé lors des visualisations du déferlement par éclairage stroboscopique). La nappe est obtenue par un double système faisceau incident barreau sphérique disposé sous le canal et traversant une partie vitrée du fond ainsi que le caisson de plage transparent.

Dans le cas d'un cylindre opaque, l'inconvénient d'un tel éclairage réside alors dans la zone d'ombre crée par le cylindre. Afin de remédier à ce désagrément, nous avons utilisé un cylindre en plastique transparent (rempli d'eau pour limiter le nombre de milieux d'indices différents traversés par la nappe laser).

Il est difficile d'obtenir des visualisations exploitables dans le domaine de l'hydrodynamique et particulièrement dans un écoulement généré par la houle. La dilution de l'ensemencement (encre, fumée, précipitation électrochimique), la gamme variée des vitesses ainsi que leur arrangement complexe impliquent l'utilisation de particules solides. Ces dernières (poudre de PVC, $\varnothing=115 \mathrm{~mm}$, 


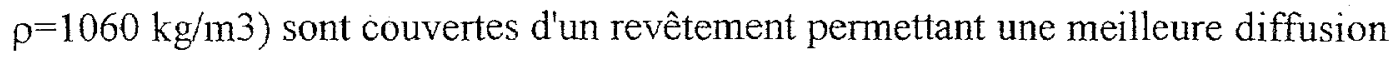
de la lumière.

Les deux photographies de la Figure 7 présentent l'écoulement autour du cylindre pour deux phases caractéristiques d'un vague limite déferlante. On met ici en évidence la nature différente du développement tourbillonnaire juste après la phase de crête (photo du haut) et pendant la phase de creux où l'on peut observer le développement d'une allée tourbillonnaire.

a)

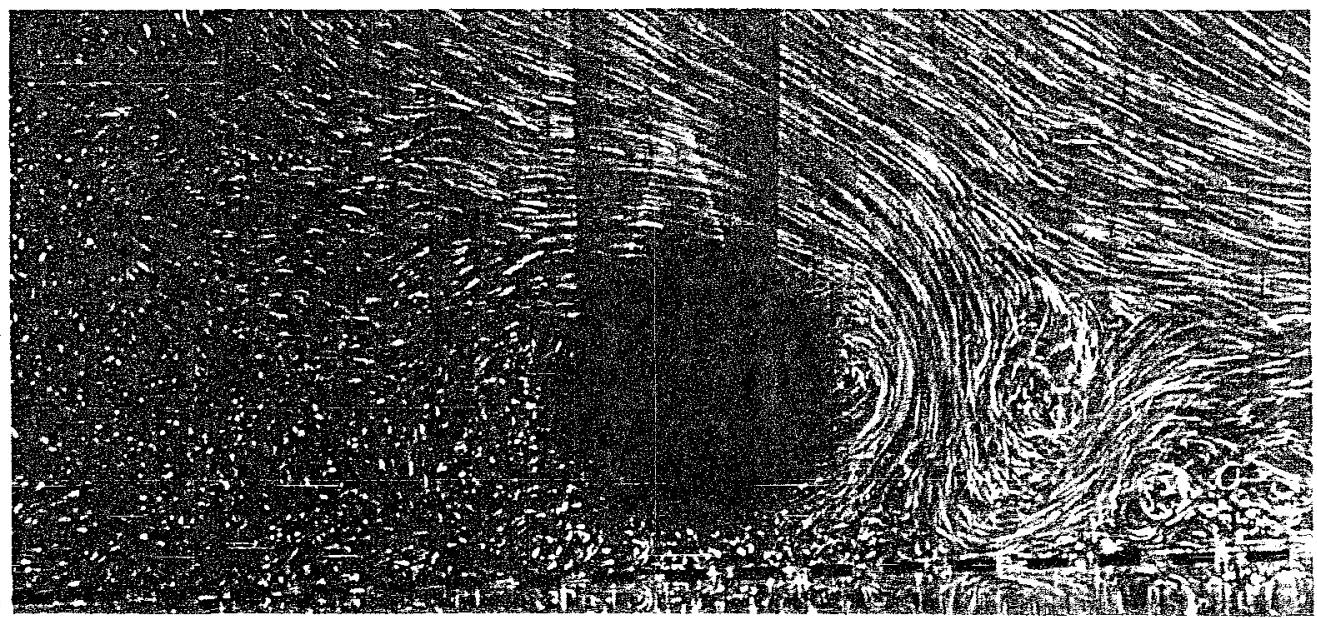

b)

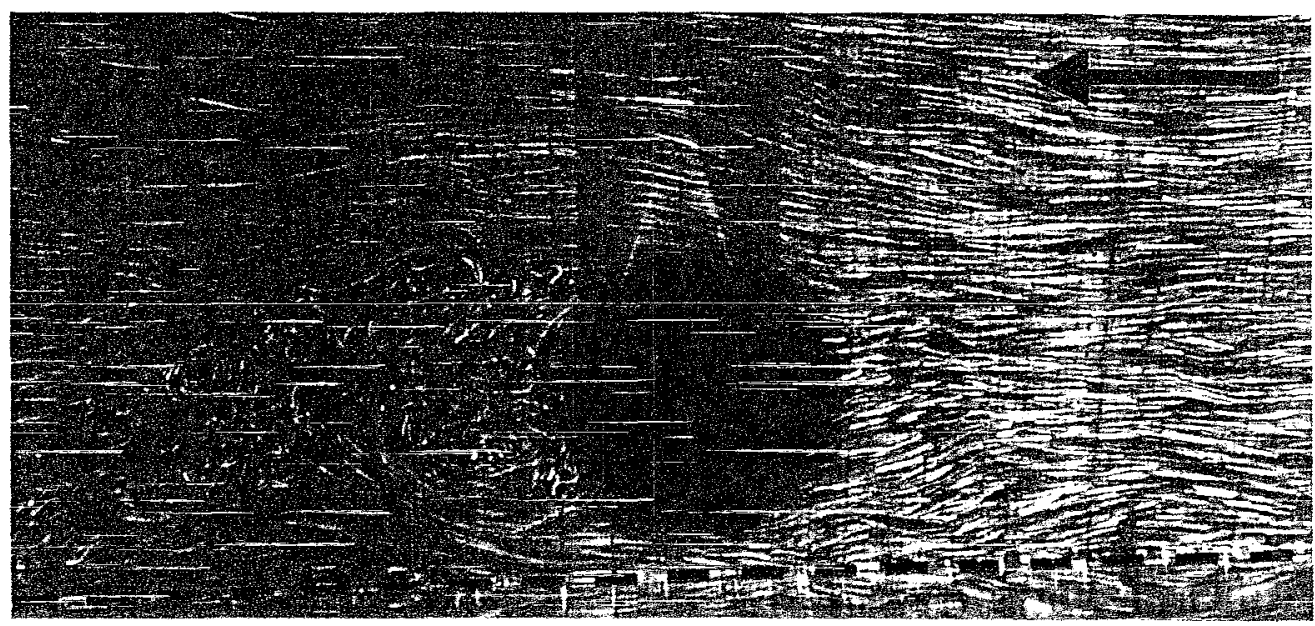

Figure 7 : a) Phase de crête b) Phase de creux - e/D $=0,125-\mathrm{KC}=22$

a) Wave crest b) wave trough $-e / D=0,125-K C=22$

\subsection{Vélocimétrie par Imagerie de Particules (PIV)}

La Vélocimétrie par Imagerie de Particules est une technique de mesure encore assez récente qui permet l'acquisition de champ complet des vitesses instantanées dans un écoulement. La PIV est encore très peu employée dans le domaine du génie côtier. Nous avons eu la possibilité d'utiliser un système Dantec PIV 2100 (Dantec, 2000) pour l'étude de l'écoulement autour d'un cylindre dans un écoulement de houle à proximité d'une paroi en pente. Cette étude vient en complément de visualisations présentées ci-dessus. 
Le principe de la PIV est assez simple. II repose sur la mesure du déplacement d'une particule entre deux instants donnés à partir de deux photographies de l'écoulement. Cette mesure du déplacement permet de déduire la vitesse (amplitude et direction) pendant l'intervalle de temps considéré. Des particules en suspension dans le fluide sont donc illuminées par une fine nappe laser. Ces particules vont alors diffuser de la lumière. Celle-ci est prise en photo par une caméra numérique. La prise de vue est répétée deux fois lors d'un court intervalle de temps ce qui conduit à deux images de l'écoulement. Ces images sont alors traitées conjointement. Par une technique de corrélation, le déplacement des différentes particules détectées entre les deux instants considérés est déterminé ce qui permet d'accéder à l'amplitude et la direction de la vitesse.

Le système Dantec est composé des éléments suivants (voir Figure 8):

- un système à deux lasers pour générer la nappe;

- une caméra numérique pour l'acquisition d'images;

- un processeur 'PIV $2100^{\prime}$ pour le traitement d'image ;

- et du logiciel 'FlowMap' installé sur un ordinateur personnel pour contrôler l'ensemble du dispositif.
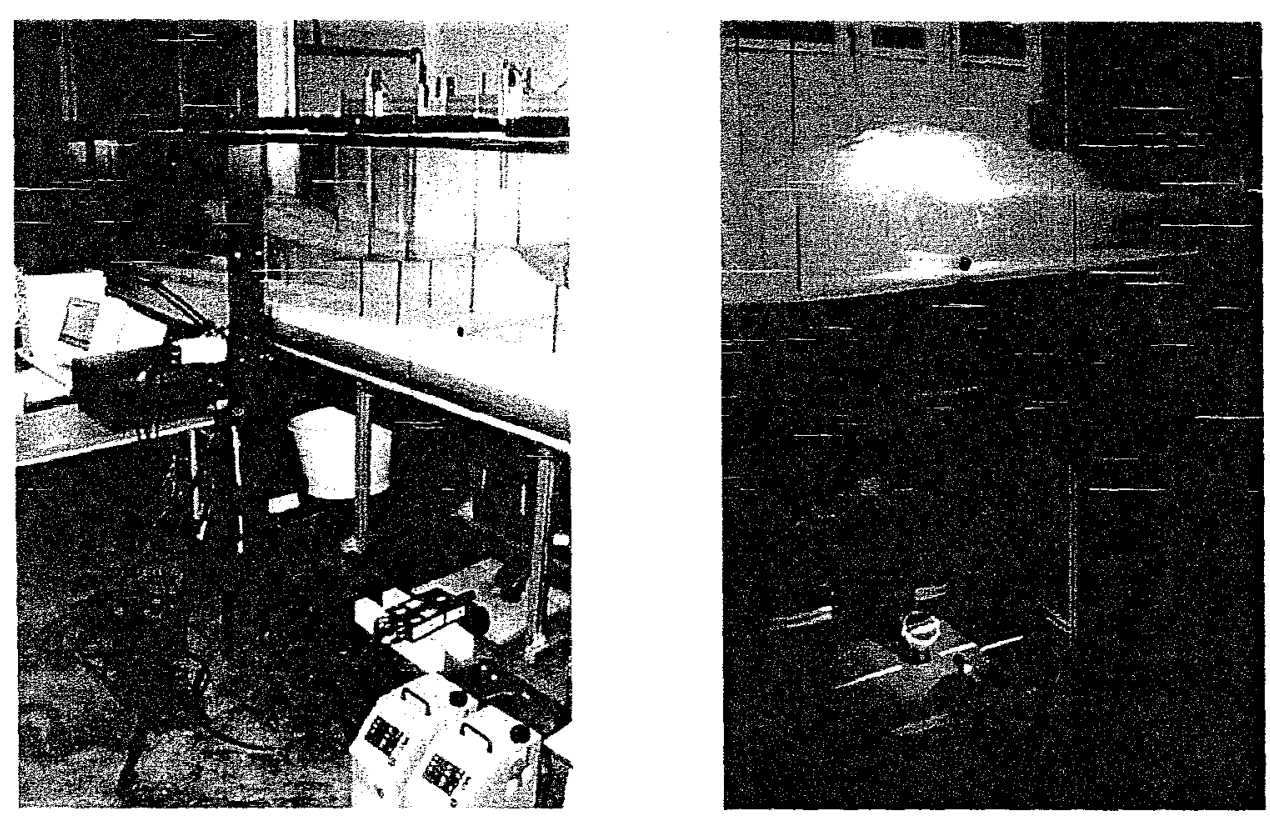

Figure 8 : Dispositif de mesure PIV

PIV measurement device

La Vélocimétrie par Imagerie de Particules est donc un instrument qui permet la réalisation de champ des vitesses $2 \mathrm{D}$ complet en un temps très court. La phase de post-traitement (affichage de champ de vecteurs, calcul de rotationnel et lignes de courant, animation) est en effet entièrement gérée par le logiciel 'FlowMap' ce qui permet une étude de l'écoulement en temps réel Cependant, le taux d'acquisition maximum $(15 \mathrm{~Hz}$ ) est un peu plus faible que celui obtenu par acquisition vidéo ou bien sûr par VDL. 
La Figure 9 présente une comparaison entre une photographie de l'écoulement et le champ des vitesses obtenu par la mesure PIV ainsi que le champ de rotationnel correspondant. La mesure PIV apporte ainsi une information quantitative concernant l'écoulement.

Un atout important de ce système est également la possibilité d'acquérir des signaux analogiques extérieurs (surface libre, efforts) pendant la mesure. Ceci peut être utile afin de mieux expliquer les phénomènes de chargement du cylindre par exemple.

a)
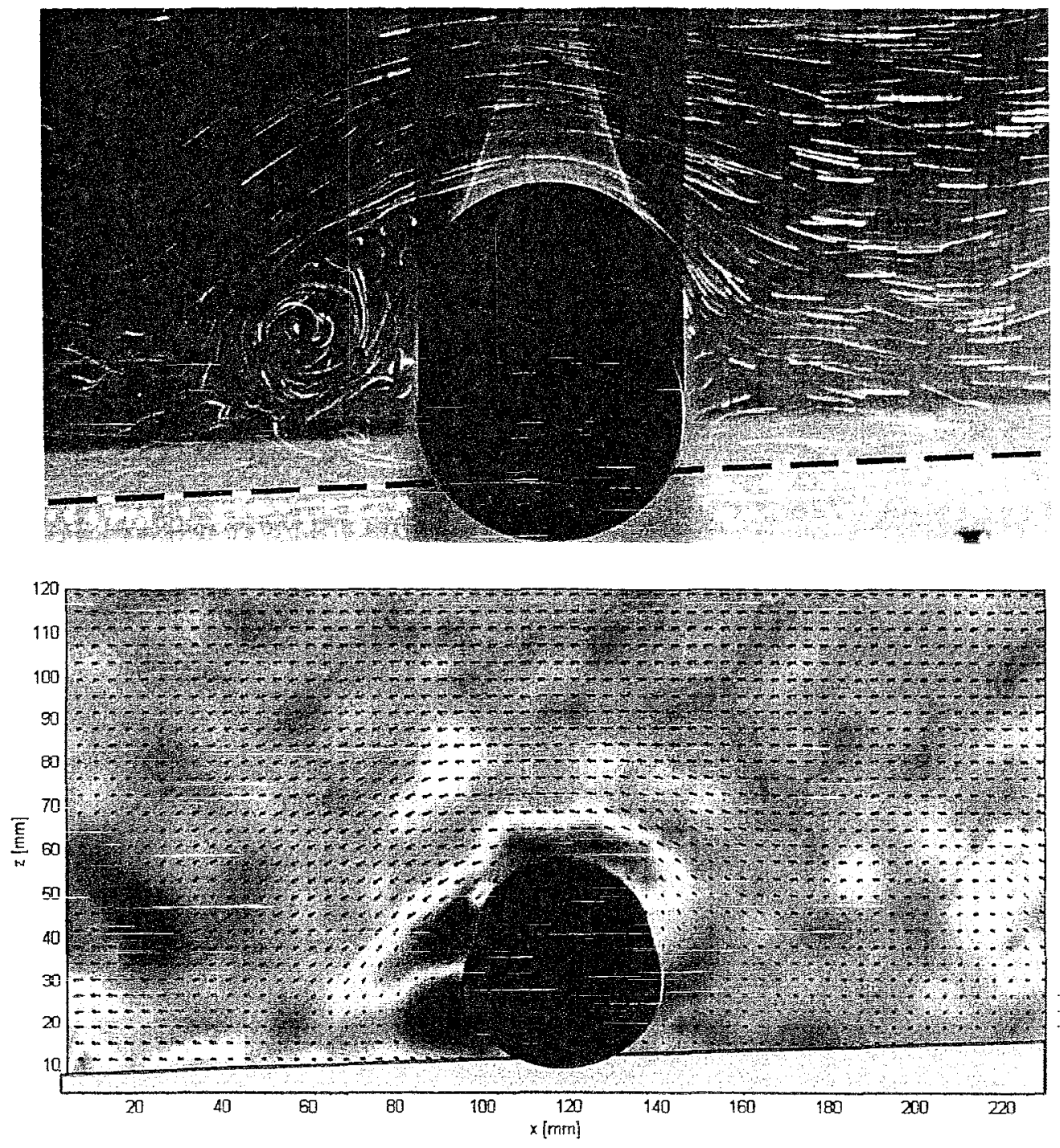

b)

Figure $9: \mathrm{KC}=22-\mathrm{e} / \mathrm{D}=0,125-\mathrm{a}$ ) photo de la phase de creux b) mesure PIV $K C=22-e / D=0,125$ - a) picture of the wave trough b) PIV measurement

\subsection{Synthèse des principaux résultats obtenus}

Ces visualisations complètent celles effectuées par ailleurs, notamment en écoulement oscillant (Sumer et al., 1991). Elles apportent également des 
enseignements inédits, notamment la dissymétrie entre les phases de flux et de reflux du fait de la nature de l'écoulement simulé (voir Figure 7). La profonde dissymétrie de la houle influence ainsi la formation, la croissance et le détachement des tourbillons. Ceux formés en phase de creux ont un temps de formation plus long et sont détachés plus nettement que ceux formés en lors du passage de la crête.

Nous avons également mis en évidence trois régimes différents d'écoulements en fonction de l'écartement relatif du cylindre par rapport au fond (cylindre posé sur le fond, à proximité du fond et éloigné du fond).

Les visualisations ont permis par ailleurs de mieux appréhender et comprendre les efforts hydrodynamiques exercés par la houle sur le cylindre en mettant en évidence l'influence des zones tourbillonnaires sur le chargement du cylindre.

\section{Remerciements}

Les auteurs remercient la société Dantec, et plus particulièrement M Tanguy, pour la démonstration du dispositif de mesures PIV effectuée au cours de l'étude de l'écoulement autour d'un cylindre.

\section{Références}

- Chevalier C. (2000) "Action de la houle sur un cylindre immergé dans la zone côtière", Thèse de Doctorat, Université de Caen, 184 p.

- Dean R. G., Dalrymple R.A. (1991) "Water wave mechanics for engineers and scientists", World Scientific, 353 p.

- Dantec Measurement Technology (2000) "Global imaging (PIV \& PLIF)", www.dantecmt.com/piv/system/

- Merzkirch W. (1987) «Flow visualization », Academic Press, $2^{\text {nd }}$ Edition, $260 \mathrm{p}$.

- Nadaoka K., Hino M., Koyano Y. (1989) "Structure of the turbulent flow field under breaking waves in the surf zone", Journal of Fluid Mechanics, Vol. 204, pp. 359-389.

- Sumer B.M., Jensen B.L., Fredsøe J. (1991) "Effect of a plane boundary on oscillatory flow around a circular cylinder", Journal of Fluid Mechanics, Vol. 225, pp. 271-300.

- Zhang D.P., Sunamura T. (1994) "Multiple bar formation by breaker-induced vortices : a laboratory approach", Proceedings of the International Conference on Coastal Engineering, Kobe 1994, pp. 2856-2870. 